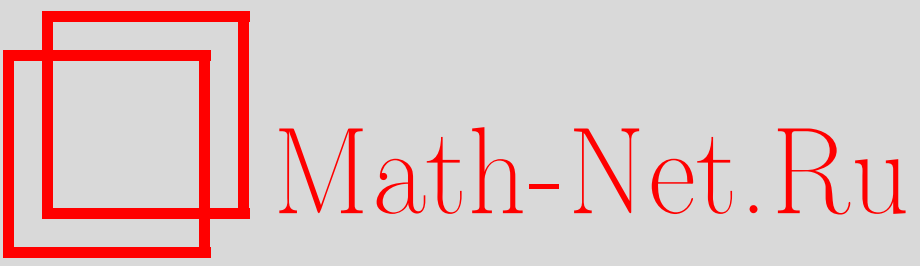

Ю. А. Боган, Задача Дирихле в двумерной стационарной анизотропной термоупругости, Вестн. Сам. гос. техн. ун-та. Сер. Физ.-мат. науки, 2010, выпуск 5(), 64-71

DOI: https://doi.org/10.14498/vsgtu820

Использование Общероссийского математического портала Math-Net.Ru подразумевает, что вы прочитали и согласны с пользовательским соглашением

http: //www. mathnet.ru/rus/agreement

Параметры загрузки:

IP : 52.90 .164 .192

26 апреля 2023 г., 02:35:50 
УДК 539.3

\title{
ЗАДАЧА ДИРИХЛЕ В ДВУМЕРНОЙ СТАЦИОНАРНОЙ АНИЗОТРОПНОЙ ТЕРМОУПРУГОСТИ
}

\section{Ю. А. Боган}

Институт гидродинамики им. М. А. Лаврентьева СО РАН, 630090, Новосибирск, пр-т Академика Лаврентьева, 15

E-mail: bogan@hydro.nsc.ru

\begin{abstract}
Изучается задача Дирихле для анизотропной термоупругой средъ. Здесъ, по определению, на границе заданы вектор перемещений и температура. Краевая задача приведена к системе интегральных уравнений. Эта система имеет слаборегулярные ядра в ограниченной области с ляпуновской границей и гельдеровыми граничными данными. Если граница области и граничные данные имеют худшие свойства гладкости, краевая задача сохраняет свойство разрешимости по Фредгольму.
\end{abstract}

Ключевые слова: интегралъные уравнения, анизотропия, упругостъ.

Введение. Интегральные уравнения редко применяются для решения стационарных задач термоупругости. Так, в статье [1] используется метод сингулярных уравнений. Не так давно автор [2] обнаружил, что при условии однозначной разрешимости краевую задачу для анизотропного материала можно легко привести к системе интегральных уравнений, если использовать простоту корней характеристического уравнения для анизотропного материала. Аналогичный подход применялся автором ранее при решении задач теории упругости. В этой работе этот подход применяется ко второй краевой задаче анизотропной термоупругости, т. е. когда на границе заданы температура и перемещения. Построена система интегральных уравнений. Если граница односвязной области достаточно гладкая, например, принадлежит классу Ляпунова, то это - система уравнений Фредгольма второго рода. Рассмотрен вопрос о гладкости напряжений.

1. Постановка задачи. Напомним необходимые сведения из термоупругости. Для определенности будем считать, что рассматривается обобщённое плоское состояние. В дальнейшем в основном используются обозначения [3]. Примем обобщённый закон Дюамеля-Неймана в следующем виде:

$$
\begin{aligned}
& \varepsilon_{11}=c_{11} \sigma_{11}+c_{12} \sigma_{22}+c_{16} \sigma_{12}+\beta_{11} u_{3}, \\
& \varepsilon_{22}=c_{12} \sigma_{11}+c_{22} \sigma_{22}+c_{26} \sigma_{12}+\beta_{22} u_{3}, \\
& \varepsilon_{12}=c_{16} \sigma_{11}+c_{26} \sigma_{22}+c_{66} \sigma_{12}-2 \beta_{66} u_{3} .
\end{aligned}
$$

Здесь $\varepsilon_{i j}, i, j \in\{1,2\}$ - деформации; $u_{1}, u_{2}$ - перемещения; $u_{3}$ - температура; $\sigma_{i j}, i, j \in\{1,2\}-$ напряжения; $c_{i j}, i, j \in\{1,2,6\}-$ податливости; $\beta_{i i}$, $i \in\{1,2,6\}$ - коэффициенты, определяющие компоненты тензора деформации свободного от внешних сил тела при изменении температуры. Напомним, чТО

$$
\varepsilon_{i j}=\frac{1}{2}\left(\frac{\partial u_{i}}{\partial x_{j}}+\frac{\partial u_{j}}{\partial x_{i}}\right), \quad i, j \in\{1,2\} .
$$

Юрий Александрович Боган (д.ф.-м.н), ведущий научный сотрудник, отдел механики деформируемого твердого тела. 
Определим из (1) напряжения и подставим их в уравнения равновесия. Получающаяся при этом система для произвольной анизотропии материала выглядит довольно громоздко, поэтому выпишем её для случая ортотропного материала:

$$
\begin{aligned}
& d_{11} \frac{\partial^{2} u_{1}}{\partial x_{1}^{2}}+d_{66} \frac{\partial^{2} u_{1}}{\partial x_{2}^{2}}+\left(d_{12}+d_{66}\right) \frac{\partial^{2} u_{2}}{\partial x_{1} \partial x_{2}}-\gamma_{1} \frac{\partial u_{3}}{\partial x_{1}}=0 \\
& \left(d_{12}+d_{66}\right) \frac{\partial^{2} u_{1}}{\partial x_{1} \partial x_{2}}+d_{66} \frac{\partial^{2} u_{2}}{\partial x_{1}^{2}}+d_{22} \frac{\partial^{2} u_{2}}{\partial x_{2}^{2}}-\gamma_{2} \frac{\partial u_{3}}{\partial x_{2}}=0
\end{aligned}
$$

Функция $u_{3}\left(x_{1}, x_{2}\right)$ - решение однородного уравнения

$$
b_{11} \frac{\partial^{2} u_{3}}{\partial x_{1}^{2}}+2 b_{12} \frac{\partial^{2} u_{3}}{\partial x_{1} \partial x_{2}}+b_{22} \frac{\partial^{2} u_{3}}{\partial x_{2}^{2}}=0
$$

в предположении, что (3) является эллиптическим: $b_{11}>0, b_{22}>0, b_{11} b_{22}-$ $-b_{12}^{2}>0$. Ясно, что простой заменой независимых переменных его можно привести к уравнению Лапласа. Однако это упрощение не всегда допустимо.

Уравнения (2), (3) можно рассматривать как систему уравнений анизотропной теории упругости, куда в качестве правых частей входят производные от температуры. Её общее решение состоит из общего решения однородной системы (температура отсутствует) и частного решения, соответствующего присутствию температурного слагаемого.

Решение будем искать в следующем виде:

$$
u_{1}=\operatorname{Re} n_{1} \varphi_{3}\left(x_{1}+\mu_{3} x_{2}\right), \quad u_{2}=\operatorname{Re} n_{2} \varphi_{3}\left(x_{1}+\mu_{3} x_{2}\right) .
$$

Здесь $\operatorname{Re} \varphi_{3}\left(x_{1}+\mu_{3} x_{2}\right)$ - решение уравнения теплопроводности, где $\operatorname{Re}$ - действительная часть комплексного выражения. При подстановке (4) в (2) получим для $n_{1}, n_{2}$ алгебраическую систему

$$
\begin{aligned}
& n_{1}\left(d_{11}+d_{66} \mu_{3}^{2}\right)+n_{2} \mu_{3}\left(d_{12}+d_{66}\right)=\gamma_{1}, \\
& n_{1} \mu_{3}\left(d_{12}+d_{66}\right)+n_{2}\left(d_{66}+d_{22} \mu_{3}^{2}\right)=\gamma_{2} \mu_{3}
\end{aligned}
$$

с определителем

$$
\delta\left(\mu_{3}\right)=\left(d_{11}+d_{66} \mu_{3}^{2}\right)\left(d_{66}+d_{22} \mu_{3}^{2}\right)-\left(d_{12}+d_{66}\right)^{2} \mu_{3}^{2} .
$$

Если $\mu_{3}$ таково, что $\delta\left(\mu_{3}\right) \neq 0$, то постоянные $n_{1}, n_{2}$ однозначно определяются из (4) и тем самым находится требуемое частное решение. Но уравнение $\delta(\mu)=0$ имеет четыре комплексно сопряжённых корня $\mu_{1}, \bar{\mu}_{1}, \mu_{2}, \bar{\mu}_{2}$, и при совпадении $\mu_{3}$ с одним из них $\delta\left(\mu_{3}\right)$ равно нулю. Следовательно, в данном случае этот подход непригоден. Тем не менее именно такой способ определения частного решения применяется в [2] без всяких оговорок.

Как представляется автору, в этом случае для определения частного решения необходимо построить фундаментальное решение системы уравнений теории упругости и свернуть его с правой частью. Так, для ортотропного материала матрица $\left(v_{i j}\right)\left(i, j \in\{1,2\}, v_{12}=v_{21}\right)$ фундаментального решения с особенностью в точке $(0,0)$ имеет следующий вид:

$$
v_{11}\left(x_{1}, x_{2}\right)=\frac{1}{2 \pi^{2}} \operatorname{Re} \sum_{k=1}^{2} \frac{a_{22} \mu_{k}^{2}+a_{66}}{N\left(\mu_{k}\right)} \ln \left(x_{1}+\mu_{k} x_{2}\right),
$$




$$
\begin{gathered}
v_{12}\left(x_{1}, x_{2}\right)=-\frac{1}{2 \pi^{2}} \operatorname{Re} \sum_{k=1}^{2} \frac{\left(a_{12}+a_{66}\right) \mu_{k}}{N\left(\mu_{k}\right)} \ln \left(x_{1}+\mu_{k} x_{2}\right), \\
v_{22}\left(x_{1}, x_{2}\right)=\frac{1}{2 \pi^{2}} \operatorname{Re} \sum_{k=1}^{2} \frac{a_{66} \mu_{k}^{2}+a_{11}}{N\left(\mu_{k}\right)} \ln \left(x_{1}+\mu_{k} x_{2}\right) .
\end{gathered}
$$

Здесь $N(\mu)=\Lambda(\mu)^{\prime}$, где $\Lambda(\mu)=d_{22} d_{66} \mu^{4}+\left(d_{11} d_{22}-d_{12}^{2}-2 d_{12} d_{66}\right) \mu^{2}+d_{11} d_{66}$. При этом $\mu_{1}, \mu_{2}$-корни уравнения $\Lambda(\mu)=0$ с положительной мнимой частью. Нетрудно видеть, что

$$
\begin{aligned}
& N\left(\mu_{1}\right)=d_{22} d_{66}\left(\mu_{1}-\mu_{2}\right)\left(\mu_{1}-\bar{\mu}_{1}\right)\left(\mu_{1}-\bar{\mu}_{2}\right), \\
& N\left(\mu_{2}\right)=d_{22} d_{66}\left(\mu_{2}-\mu_{1}\right)\left(\mu_{2}-\bar{\mu}_{1}\right)\left(\mu_{2}-\bar{\mu}_{2}\right) .
\end{aligned}
$$

Положим

$$
v_{k}\left(x_{1}, x_{2}\right)=\int_{Q} \sum_{s=1}^{2} v_{k s}\left(x_{1}-t_{1}, x_{2}-t_{2}\right) f_{s}\left(t_{1}, t_{2}\right) d t_{1} d t_{2}, \quad k \in\{1,2\} .
$$

Если при этом

$$
f_{1}=\gamma_{1} \frac{\partial u_{3}}{\partial x_{1}}, \quad f_{2}=\gamma_{2} \frac{\partial u_{3}}{\partial x_{2}},
$$

то функции $v_{k}\left(x_{1}, x_{2}\right)(k \in\{1,2\})$ дают требуемое частное решение системы уравнений.

В реальной физической ситуации упругие постоянные и температурные параметры известны только с некоторой погрешностью, и поэтому при малом изменении параметров можно придти к неравенству $\delta\left(\mu_{3}\right) \neq 0$, справедливость которого будет предполагаться в дальнейшем. Отсюда для перемещений и температуры получим представление через аналитические функции (с точностью до жёсткого перемещения):

$$
\begin{aligned}
& u_{1}\left(x_{1}, x_{2}\right)=\operatorname{Re}\left\{b_{11} \varphi_{1}\left(z_{1}\right)+b_{12} \varphi_{2}\left(z_{2}\right)+n_{1} \varphi_{3}^{\prime}\left(z_{3}\right)\right\} \\
& u_{2}\left(x_{1}, x_{2}\right)=\operatorname{Re}\left\{b_{21} \varphi_{1}\left(z_{1}\right)+b_{22} \varphi_{2}\left(z_{2}\right)+n_{2} \varphi_{3}^{\prime}\left(z_{3}\right)\right\} \\
& u_{3}\left(x_{1}, x_{2}\right)=\operatorname{Re} \varphi_{3}\left(z_{3}\right)
\end{aligned}
$$

где $b_{1 k}=c_{11} \mu_{k}^{2}+c_{12}-c_{16} \mu_{k}, b_{2 k}=c_{12} \mu_{k}+c_{22} \mu_{k}^{-1}-c_{26}, k \in\{1,2\}$.

2. Задача Дирихле. Количество постановок краевых задач термоупругости, допускающих приведение к регулярным интегральным уравнениям, довольно велико. Рассмотрим одну из них, которую будем называть задачей Дирихле: будем предполагать, что на границе заданы вектор перемещений и температура. Пусть $Q_{i}$ - односвязная ограниченная область на плоскости со спрямляемой жордановой границей $\partial Q$ длины $M$ класса $C^{1, \alpha}(0, M) ; s-$ длина дуги, отсчитываемая от фиксированной точки границы. В дальнейшем утверждение $\partial Q \in C^{l, \alpha}(\partial Q)$ означает, что функции $x_{1}(s), x_{2}(s) \in C^{l, \alpha}(0, M)$. Под $C^{l, \alpha}(\Omega)$ в области или на отрезке $\Omega$ понимается, как обычно, банахово пространство функций, имеющих $l$ непрерывных производных в области $\Omega$, 
причём производная порядка $l$ удовлетворяет условию Гельдера с показателем $\alpha, 0<\alpha<1$. Через $Q_{e}$ обозначим область, внешнюю по отношению к $Q_{i}$.

Для определения перемещений необходимо сначала решить задачу Дирихле для уравнения теплопроводности. Положим $t_{k}(s)=x_{1}(s)+\mu_{k}(s), t_{k 0}=$ $=x_{1}\left(s_{0}\right)+\mu_{k}\left(s_{0}\right), k \in\{1,2,3\}$. Будем искать решение уравнения (3) в таком виде: $u\left(x_{1}, x_{2}\right)=\operatorname{Re} \varphi_{3}\left(x_{1}+\mu_{3} x_{2}\right)$. Здесь $\mu_{3}=\gamma+i \beta(\beta>0)$ определяется из уравнения $b_{11}+2 b_{12} \mu+b_{22} \mu^{2}=0$ и отлично от нуля, так как $b_{11} b_{22}-b_{12}^{2}>0$ ввиду эллиптичности уравнения (3).

Рассмотрим задачу Дирихле $D_{i}$ :

$$
\left.u_{3}\left(x_{1}, x_{2}\right)\right|_{\partial Q}=g_{3}\left(s_{0}\right), \quad g_{3}\left(s_{0}\right) \in C^{0, \alpha}(\partial Q) .
$$

Представим $u_{3}\left(x_{1}, x_{2}\right)$ в виде действительной части интеграла типа Коши с вещественной плотностью $f_{3}(s)$ :

$$
u_{3}\left(x_{1}, x_{2}\right)=\operatorname{Re} \frac{1}{\pi i} \int_{\partial Q} \frac{f(s) d t_{3}}{t_{3}-z_{3}} .
$$

Здесь и в дальнейшем $d t_{j}=\left(x_{1}^{\prime}(s)+\mu_{j} x_{2}^{\prime}(s)\right) d s, j \in\{1,2,3\}$. В силу формулы Сохоцкого, когда точка $z \in Q$ стремится к точке $t_{0}=x_{1}\left(s_{0}\right)+i x_{2}\left(s_{0}\right) \in \partial Q$ $\left(s_{0} \in(\partial Q)\right)$ изнутри области, имеем

$$
\lim _{z \rightarrow t_{0}} \frac{1}{\pi i} \int_{\partial Q} \frac{g_{j} d t_{j}}{t_{j}-z_{j}}=g_{j}\left(s_{0}\right)+\frac{1}{\pi i} \int_{\partial Q} \frac{g_{j} d t_{j}}{t_{j}-t_{j 0}} .
$$

Напомним, что формула Сохоцкого применима, если $g_{j}(s) \in C^{0, \alpha}(\partial Q)$. Тогда получим на границе интегральное уравнение для определения неизвестной плотности $f_{3}(s)$ :

$$
f_{3}\left(s_{0}\right)+\operatorname{Re} \frac{1}{\pi i} \int_{\partial Q} \frac{f_{3}(s) d t_{3}}{t_{3}-t_{30}}=g_{3}\left(s_{0}\right), \quad g_{3}\left(s_{0}\right) \in C^{0, \alpha}(\partial Q) .
$$

То, что (7) является уравнением Фредгольма второго рода, следует из следующего рассуждения. Достаточно доказать, что ядро интегрального оператора в (7) имеет слабую особенность. Действительно, при помощи замены независимой переменной $x=x_{1}+\gamma x_{2}, y=\beta x_{2}, \mu=\gamma+i \beta(\beta>0)$ получим, что

$$
\operatorname{Re} \frac{1}{\pi i} \int_{\partial Q} \frac{f(s) d t_{3}}{t_{3}-z_{3}}=\operatorname{Re} \frac{1}{\pi i} \int_{\partial Q} \frac{f_{3}(s) d t}{t-z}
$$

где $t=x(s)+i y(s), z=x+i y$. Правая часть в этом соотношении, очевидно, совпадает с потенциалом двойного слоя для уравнения Лапласа. Действительно, при замене $\xi_{1}=x_{1}+\gamma x_{2}, \xi_{2}=\beta x_{2}$ уравнение (3) обращается в уравнение Лапласа. Как показано в [1], его ядро имеет слабую особенность, если граница имеет нормальный вектор, удовлетворяющий условию Гельдера, т. е. если функции $x(s), y(s)$, задающие форму границы, подчинены условию

$$
\left|x_{k}^{\prime}(s)-x_{k}^{\prime}\left(s_{0}\right)\right| \leqslant c\left|s-s_{0}\right|^{\alpha}, \quad 0<\alpha<1, \quad k \in\{1,2\} .
$$


Напомним, что, как хорошо известно [1], конечным числом итераций ядро со слабой особенностью можно превратить в непрерывное, и поэтому к уравнению (7) можно применить теорию Фредгольма.

3. Интегральные уравнения. Рассмотрим следующую краевую задачу: определить поле перемещений и температуру по их значениям на границе:

$$
\begin{gathered}
\left.u_{k}\left(x_{1}, x_{2}\right)\right|_{\partial Q}=g_{k}(s), \quad g_{k}(s) \in C^{0, \alpha}(\partial Q), \quad k \in\{1,2\}, \\
\left.u_{3}\left(x_{1}, x_{2}\right)\right|_{\partial Q}=g_{3}(s), \quad g_{3}(s) \in C^{1, \alpha}(\partial Q) .
\end{gathered}
$$

Назовём её для краткости задачей Дирихле для системы уравнений термоупругости. Тогда функции $\varphi_{1}\left(z_{1}\right), \varphi_{2}\left(z_{2}\right)$ определяются из граничных условий

$$
\begin{gathered}
\operatorname{Re}\left\{b_{11} \varphi_{1}\left(z_{1}\right)+b_{12} \varphi_{2}\left(z_{2}\right)\right\}=g_{1}\left(s_{0}\right)-n_{1} \varphi_{3}^{\prime}\left(t_{3}\left(s_{0}\right)\right), \\
\operatorname{Re}\left\{b_{21} \varphi_{1}\left(z_{1}\right)+b_{22} \varphi_{2}\left(z_{2}\right)\right\}=g_{2}\left(s_{0}\right)-n_{2} \varphi_{3}^{\prime}\left(t_{3}\left(s_{0}\right)\right), \\
\operatorname{Re} \varphi_{3}\left(z_{3}\right)=g_{3}\left(s_{0}\right), \quad g_{3}(s) \in C^{1, \alpha}(\partial Q) .
\end{gathered}
$$

Положим $\chi_{k}(s)=g_{k}(s)-n_{k} \varphi^{\prime}\left(t_{3}(s)\right), k \in\{1,2\}$. Тогда функции $\chi_{k}(s) \in$ $\in C^{0, \alpha}(\partial Q)$. Функция $f_{3}(s)$ - плотность интеграла типа Коши для определения температуры - определяется из уравнения (7). При этом поле перемещений должно иметь первые производные всюду в области $Q$, интегрируемые с квадратом в замкнутой области $\bar{Q}$, так как иначе краевая задача не будет иметь единственного решения. Будем искать аналитические функции $\varphi_{k}\left(z_{k}\right)$ в виде интегралов типа Коши:

$$
\varphi_{k}\left(z_{k}\right)=\frac{1}{\pi i} \int_{\partial Q} \frac{\omega_{k}(s) d t_{k}}{t_{k}-z_{k}}, \quad k \in\{1,2,3\} .
$$

Здесь $t_{k}=x_{1}(s)+\mu_{k} x_{2}(s), k \in\{1,2\}$. Пусть $f_{1}(s), f_{2}(s)$ - некоторые вещественные функции. Решим систему уравнений

$$
b_{11} \omega_{1}+b_{12} \omega_{2}=f_{1}(s), \quad b_{21} \omega_{1}+b_{22} \omega_{2}=f_{2}(s)
$$

методом Крамера и подставим её решение в (6). Тогда перемещения будут записаны в следующем виде:

$$
\begin{aligned}
& u_{1}\left(x_{1}, x_{2}\right)=\operatorname{Re}\left\{\frac{b_{11}}{\pi i \delta} \int_{\partial Q} \frac{\left(b_{22} f_{1}(s)-b_{12} f_{2}(s)\right) d t_{1}}{t_{1}-z_{1}}+\right. \\
& \left.+\frac{b_{12}}{\pi i \delta} \int_{\partial Q} \frac{\left(-b_{21} f_{1}(s)+b_{11} f_{2}(s)\right) d t_{2}}{t_{2}-z_{2}}\right\}, \\
& u_{2}\left(x_{1}, x_{2}\right)=\operatorname{Re}\left\{\frac{b_{21}}{\pi i \delta} \int_{\partial Q} \frac{\left(b_{22} f_{1}(s)-b_{12} f_{2}(s)\right) d t_{1}}{t_{1}-z_{1}}+\right.
\end{aligned}
$$




$$
\left.+\frac{b_{22}}{\pi i \delta} \int_{\partial Q} \frac{\left(-b_{21} f_{1}(s)+b_{11} f_{2}(s)\right) d t_{2}}{t_{2}-z_{2}}\right\} .
$$

Здесь $\delta=b_{11} b_{22}-b_{12} b_{21}$ - определитель системы (8). Нетрудно видеть, что он пропорционален разности $\mu_{1}-\mu_{2}$ и поэтому можно записать, что $\delta=\xi \cdot\left(\mu_{1}-\right.$ $\left.-\mu_{2}\right)$. При этом $\xi$ зависит от симметрических функций, от корней характеристического уравнения, и, как следствие, от коэффициентов обобщённого закона Гука. В дальнейшем предполагается, что $\xi \neq 0$. В краевых задачах теории упругости он всегда отличен от нуля ввиду положительной определённости удельной потенциальной энергии деформации. Например, для ортотропного материала $\left(c_{16}=0, c_{26}=0\right)$ составляет величину

$$
\xi=\left(\gamma_{1} \gamma_{2}\right)^{-1}\left\{\left(c_{11} c_{22}-c_{12}^{2}\right) \sqrt{\frac{c_{22}}{c_{11}}}+c_{22} c_{66}\right\}
$$

и положителен, так как $c_{11} c_{22}-c_{12}^{2}>0, c_{11}>0, c_{22}>0, c_{66}>0$. При этом для изотропного материала $\xi=(1+\nu)(3-\nu) E^{-2}$, где $\nu$ - коэффициент Пуассона, $E$ - модуль Юнга.

Нетрудно видеть, что перемещения можно представить и так:

$$
\begin{aligned}
& \begin{aligned}
& u_{1}\left(x_{1}, x_{2}\right)=\operatorname{Re} \frac{1}{\pi i} \int_{\partial Q} \frac{f_{1}(s) d t_{1}}{t_{1}-z_{1}}+ \\
&+\operatorname{Re} \frac{b_{12}}{\pi i \delta} \int_{\partial Q}\left(-b_{21} f_{1}(s)+b_{11} f_{2}(s)\right)\left(\frac{d t_{2}}{t_{2}-z_{2}}-\frac{d t_{1}}{t_{1}-z_{1}}\right), \\
& u_{2}\left(x_{1}, x_{2}\right)=\operatorname{Re} \frac{1}{\pi i} \int_{\partial Q} \frac{f_{2}(s) d t_{2}}{t_{2}-z_{2}}+ \\
& \quad+\operatorname{Re} \frac{b_{21}}{\pi i \delta} \int_{\partial Q}\left(b_{22} f_{1}(s)-b_{12} f_{2}(s)\right)\left(\frac{d t_{1}}{t_{1}-z_{1}}-\frac{d t_{2}}{t_{2}-z_{2}}\right) .
\end{aligned}
\end{aligned}
$$

Применяя формулу Сохоцкого, получим систему интегральных уравнений на границе

$$
\begin{aligned}
& f_{1}\left(s_{0}\right)+\operatorname{Re} \frac{1}{\pi i} \int_{\partial Q} \frac{f_{1}(s) d t_{1}}{t_{1}-t_{10}}+ \\
& +\operatorname{Re} \frac{b_{12}}{\pi i \delta} \int_{\partial Q}\left(-b_{21} f_{1}(s)+b_{11} f_{2}(s)\right)\left(\frac{d t_{2}}{t_{2}-t_{20}}-\frac{d t_{1}}{t_{1}-t_{10}}\right)=\chi_{1}\left(s_{0}\right), \\
& f_{2}\left(s_{0}\right)+\operatorname{Re} \frac{1}{\pi i} \int_{\partial Q} \frac{f_{2}(s) d t_{2}}{t_{2}-t_{20}}+
\end{aligned}
$$




$$
+\operatorname{Re} \frac{b_{21}}{\pi i \delta} \int_{\partial Q}\left(b_{22} f_{1}(s)-b_{12} f_{2}(s)\right)\left(\frac{d t_{1}}{t_{1}-t_{10}}-\frac{d t_{2}}{t_{2}-t_{20}}\right)=\chi_{2}\left(s_{0}\right) .
$$

Доказательство регулярности этой системы уравнений аналогично данному в работе [2] и потому здесь не приводится.

4. Гладкость решения. Решение задачи Дирихле имеет весьма ограниченную гладкость. Действительно, если граничные данные принадлежат только классу $C^{0, \alpha}(\partial Q)$, то функции

$$
\left|\varphi_{k}^{\prime}\left(z_{k}\right)\right| \leqslant C|\delta(\partial Q)|^{\alpha-1}, \quad k \in\{1,2,3\},
$$

где $\delta(\partial Q)$ - расстояние до границы области [4, стр. 69]. Эта оценка, в частности, показывает, что производные от перемещений интегрируемы с квадратом, только если $\alpha>1 / 2$. Поэтому решение задачи Дирихле будет единственным, только если $\alpha>1 / 2$. Справедливость предыдущей оценки легко проверить для решения краевой задачи в полуплоскости. Пусть $\varphi\left(z_{3}\right)$, например, решение задачи теплопроводности в полуплоскости $\mathbb{R}_{+}{ }^{2}=\left\{\left(x_{1}, x_{2}\right) ; x_{2}>0\right\}$. Тогда $\varphi_{3}\left(z_{3}\right)$ представима интегралом типа Коши:

$$
\varphi_{3}\left(z_{3}\right)=\frac{1}{\pi i} \int_{-\infty}^{\infty} \frac{\mu(t) d t}{t-z_{3}}
$$

где $\mu(t) \in C^{0, \alpha}$ на любом подынтервале вещественной оси. Положим для сокращения записи $z=z_{3}, z=x+i y$, т. е. $\mu=i$ (это не влияет на существо оценки). Тогда

$$
y \varphi^{\prime}(z)=\frac{1}{\pi i} \int_{-\infty}^{\infty} \frac{y \mu(t)}{(t-z)^{2}} d t .
$$

При учёте неравенств $|z-x|=y \leqslant|t-z|,|t-x| \leqslant|t-z|$, справедливых при любом вещественном $t$, получим следующую цепочку неравенств:

$$
\begin{aligned}
\left|y \varphi^{\prime}(z)\right| \leqslant A \int_{-\infty}^{\infty} \frac{y d t}{|t-z|^{2-\alpha}} \leqslant A y^{1+\alpha} & \int_{-\infty}^{\infty}\left|\frac{y}{t-z}\right|^{-1-\alpha} \frac{d t}{|t-z|^{2}} \leqslant \\
& \leqslant A y^{1+\alpha} \int_{-\infty}^{\infty} \frac{d t}{|t-z|^{2}}=\pi A y^{\alpha}, \quad A>0
\end{aligned}
$$

Аналогичное рассуждение применялось в статье [5]. При этом, как отмечалось в [2], для интегрируемости напряжений с квадратом необходимо предполагать, что показатель Гельдера $\alpha>1 / 2$. Для непрерывности напряжений на границе следует предполагать принадлежность граничных данных классам $g_{k}(s) \in C^{1, \alpha}(\partial Q), k \in\{1,2\}, g_{3}(s) \in C^{2, \alpha}(\partial Q)$.

\section{БИБЛИОГРАФИЧЕСКИЙ СПИСОК}

1. Zhao Yu-Qui On the Plane Orthotropic Stress Problem of Quasi-Static Thermoelasticity // J. Elasticity, 1997. - Vol.46, No. 3. - P. 199-216.

2. Боган Ю. А. Регулярные интегральные уравнения для второй краевой задачи в анизотропной теории упругости // Изв. РАН. МTT, 2005. - № 4. - С. 17-26.

3. Прусов И. А. Термоупругие анизотропные пластинки. - Минск: БГУ, 1978. - 200 с. 
4. Мусхелишвили Н. И. Сингулярные интегральные уравнения. - М.: Наука, 1968. - 511 с.

5. Бикчантаев И. А. Краевая задача для однородного эллиптического уравнения с постоянными коэффициентами // Изв. вузов. Матем., 1975. - №6. - С. 3-13.

Поступила в редакцию 18/VIII/2010;

в окончательном варианте - 22/IX/2010.

MSC: 74B05, 74E10

\section{THE DIRICHLET PROBLEM IN THE 2D STATIONARY} ANISOTROPIC THERMOELASTICITY

\section{Yu. A. Bogan}

M. A. Lavrentyev Institute of Hydrodynamics, Siberian Branch of RAS,

15, Lavrentyeva pr., Novosibirsk, 630090, Russia

E-mail: bogan@hydro.nsc.ru

In this article the Dirichlet problem for an anisotropic thermoelastic media is studied. It means, by definition, that a displacement vector and a stationary temperature are assigned at a boundary. This boundary value problem is reduced to a system of integral equations. Kernels of integral operators, entering into this system, are weakly regular in a bounded region with a Lyapunov boundary and Hölder continuous boundary data. This boundary value problem keeps up the property of Fredholm solvability if a region and boundary data have weaker properties of smoothness.

Key words: integral equations, anisotropy, elasticity.

Original article submitted 18/VIII/2010; revision submitted 22/IX/2010.

Yurii A. Bogan, Dr. Sci. (Phys. \& Math.), Leading Research Scientist, Dept. of Deformable Solid Body. 